\title{
Mathematical Modeling of the Impact of Hospital Occupancy: When Do Dwindling Hospital Beds Cause ED Gridlock?
}

\author{
Lori Whelan, Boyd Burns, Michael Brantley, Tyler Haas, \\ Annette O. Arthur, and Stephen H. Thomas \\ University of Oklahoma College of Medicine and Hillcrest Medical Center, Tulsa, OK 74135, USA \\ Correspondence should be addressed to Annette O. Arthur; annette-arthur@ouhsc.edu
}

Received 24 April 2014; Revised 25 June 2014; Accepted 25 June 2014; Published 15 July 2014

Academic Editor: Angelo P. Giardino

Copyright (c) 2014 Lori Whelan et al. This is an open access article distributed under the Creative Commons Attribution License, which permits unrestricted use, distribution, and reproduction in any medium, provided the original work is properly cited.

\begin{abstract}
Objectives. The time emergency department (ED) patients spend from presentation to admittance is known as their length of stay (LOS). This study aimed to quantify the inpatient occupancy rate (InptOcc)/ED LOS relationship and develop a methodology for identifying resource-allocation triggers using InptOcc-LOS association-curve inflection points. Methods. This study was conducted over 200 consecutive days at a 700-bed hospital with an annual ED census of approximately 50,000 using multivariate spline (piecewise) regression to model the InptOcc/LOS relationship while adjusting for confounding covariates. Nonlinear modeling was used to assess for InptOcc/LOS associations and determine the inflection point where InptOcc profoundly impacted LOS. Results. At lower InptOcc, there was no association. Once InptOcc reached $\geq 88 \%$, there was a strong InptOcc/LOS association; each $1 \%$ InptOcc increase predicted a 16-minute (95\% CI, 12-20 minutes) LOS prolongation, while the confounder-adjusted analysis showed each $1 \%$ InptOcc increase $>89 \%$ precipitating a 13 -minute $(95 \%$ CI, $10-16$ minutes) LOS prolongation. Conclusions. The study hospital's InptOcc was a significant predictor of prolonged ED LOS beyond the identified inflection point. Spline regression analysis identified a clear inflection point in the InptOcc-LOS curve that potentially identified a point at which to optimize inpatient bed availability to prevent increased costs of prolonged LOS.
\end{abstract}

\section{Background}

One of the most important emergency department (ED) operations parameters is the time that patients who are ultimately admitted to the hospital spend in the ED from presentation to transfer to a hospital bed. This parameter, the ED length of stay for admitted patients (hereafter, LOS), has both direct and indirect importance.

LOS' intrinsic importance is related to the fact that shorter LOS translates into improved effective ED care capacity: more patients can be seen in a given timeframe if patient LOS is decreased. In addition to its intrinsic importance, LOS is also important for indirect reasons (e.g., improved patient satisfaction, lower morbidity/mortality, and overall costs of care) $[1,2]$. A recent overview of the literature addressing ED operations and performance measures concluded that LOS and related time frames were the most recommended performance measures to follow quality, efficiency, and sustainability of ED operations [3].
A critical consideration of LOS is its impact on the rate of patients who left-without-being-seen (LWBS). Previous work has confirmed that at our hospital (as is the case at many other facilities), LOS is one of the statistically and operationally most important predictors of LWBS $[4,5]$. For example, at one hospital in Chicago, decreasing LOS had an effect of relative decrease in LWBS of roughly 33\% [6]. Other investigations in myriad ED settings (e.g., pediatric ED and psychiatric ED) confirm the influence of time efficiency on LWBS $[7,8]$.

While many factors impact LOS, one parameter of particular interest is the inpatient hospital occupancy rate (hereafter, InptOcc). At our hospital, InptOcc is reported daily by taking an average of the hospital's occupied acutecare beds over the past 24 hours. The perception of the ED administrative group that InptOcc was closely related to highLOS days in the ED led to the current study's execution.

Previous investigations of ED operations have included emphasis on the fact that resources for managing the crowded 
ED — and thus resources for improving LOS — are limited. The logistical utility of identifying a trigger point for resource allocation (e.g., extra staffing, assembly of bed huddle meetings to work on urgently improving inpatient capacity) is that the "InptOcc trigger point" would serve as a data-driven indicator to justify resource allocation to address a major root cause (high InptOcc) of prolonged LOS.

While inpatient efficiency is of great importance, hospitals should also respond quickly to operational stresses. It is this "reserve resource allocation" that is the focus of the costbenefit case for identification of a trigger point in InptOcc. It is not economical to deploy maximal resources around the clock; using InptOcc triggers as an impetus for resource allocation (e.g., calling in nurses to open new hospital beds) has the potential to optimize resource use and right size the associated expenditures.

Given the importance of LOS, the current study was designed to analyze data from a single ED to identify whether the perceived impact of InptOcc on LOS was in fact accurate. A second aim in the event InptOcc which was associated with risk of prolonged LOS was to determine whether there is a "cutpoint" at which the relationship between InptOcc and LOS became particularly pronounced. To find this cutpoint, the linearity of the InptOcc-LOS association was assessed after adjusting for potential covariates to determine whether there was a specific point at which there was marked increase in the slope of the line depicting the InptOcc-LOS association. Identification of such a cutpoint would allow for planning to optimize resource allocation when the InptOcc approaches the trigger point.

\section{Methods}

2.1. Study Design, Interventions, and Time Frame. This was a prospective study, entailing definition of the study question prior to design of the data collection process. The study intervention was the implementation, in September 2013, of new ED patient tracking software that allowed for precise and accurate measurement of the parameters to be analyzed. The study timeframe was the 200 consecutive days of data collection commencing with the date of software implementation in September 2013.

While the study itself did not entail specific implementation of interventions, the study hospital does have ongoing operational improvement efforts that focus on both the inpatient and ED settings. We analyzed the surrogate covariate of time to assess confounding of the aforementioned efforts. We then used the assessment for association between the time period of the study and the outcome of interest as a coarse method of adjustment for these hospital-based operations improvement efforts.

As this was an observational study of an administrative database that did not entail review of any patient information, the study was exempt from review by the hospital ethics board.

2.2. Data Collected. The unit of analysis was the day. The data collected for use in this analysis included the major dependent variable of LOS and the major independent variable of InptOcc. LOS was defined as the mean daily LOS for ED patients who were admitted to the hospital. InptOcc was defined as a percentage occupancy of the hospital's acutecare beds as reported at 0700 each morning (e.g., if $2 \%$ of the hospital's acute-care beds were open on average during the 24 hours prior to daily reporting, the InptOcc parameter's value for that study day would be $98 \%$ ).

Patient parameters collected as part of this study included daily summaries of triage acuity (measured on a 5-point scale with 1 being the highest and 5 being the lowest acuity) and ED admission rate.

Additional parameters assessed as potential confounders included the number of patients presenting to the ED on a given day (ED census) and the day of the week. As mentioned above, the study time frame (within the period of 200 days) was also assessed for potential confounding by varying operations improvement efforts that are ongoing at the study hospital and ED. The study timeframe was assessed in quarters of 50 days each.

2.3. Statistical Analysis. Skewness-kurtosis testing was used to assess normality of the data. For normal data, the descriptive statistics included mean \pm standard deviation (SD), with 95\% confidence interval (CI) reported for the mean. For nonnormal data central tendency was reported as median with interquartile range (IQR).

After graphic depiction of a curvilinear relationship between InptOcc and LOS, nonlinear modeling was used to identify the best "knot" in the spline (also known as piecewise) regression function. In other words, spline regression was used to determine the "cutpoint" in InptOcc, at which LOS times began to increase substantially.

The knot-finding regression approach used followed the method of Mitchell [9], as described for use with the statistical software used for all analysis in this study (Stata 13MP, StataCorp, College Station, TX). The approach used was that for analyzing data with an unknown knot (i.e., unknown whether a knot exists in the regression line and unknown where that knot might be if it does exist).

The spline logistic regression modeling was multivariate, adjusting for factors such as ED census, day of week, and patient acuity (triage severity as well as admission percentage). In order to account for skewness in the continuous variables assessed, robust standard errors were calculated. Univariate regression was used to assess for potential significance of association between potential confounders and the dependent variable of interest (LOS). As model-building proceeded, potential confounders were reintroduced into the model for assessment as per standard approaches of assessing for $>20 \%$ change in $\beta$ point estimate, regardless of statistical significance [10].

\section{Results}

3.1. Descriptive Statistics. Analysis of the 200 days' data with the skewness-kurtosis test revealed the following variables to be normally distributed: ED census, admission percentage, and triage acuity. The LOS data and InptOcc data were 
TABLE 1: Overall characteristics for study period of 200 days.

\begin{tabular}{lc}
\hline ED census (mean \pm SD) & $124 \pm 16$ \\
$\begin{array}{l}\text { Left-without-being-seen \% (median, IQR) } \\
\text { Length of stay (LOS) minutes (median, } \\
\text { interquartile range) }\end{array}$ & 0.9 (0 to 1.8) \\
$\begin{array}{l}\text { \% higher acuity triage (class 1, 2, or 3) } \\
\text { \% Inpatient occupancy (median, } \\
\text { interquartile range) }\end{array}$ & 339 (236 to 594) \\
\hline
\end{tabular}

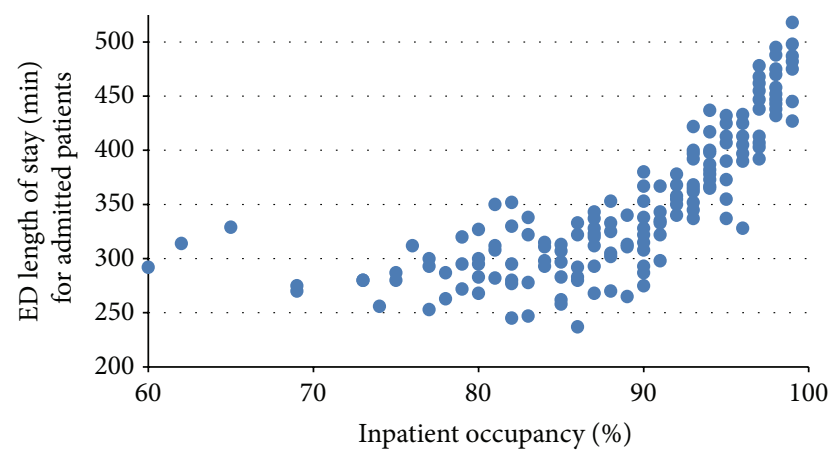

FIGURE 1: Univariate regression plot, ED length of stay (LOS) predicted by inpatient occupancy (InptOcc).

nonnormal. The descriptive analysis of the results for the $n=200$ study days is reported in Table 1 .

3.2. Analytic Statistics. The study hypothesis being tested with these results had two parts. Part one of the hypothesis was that up to a certain point, increasing InptOcc has little or no effect on LOS for admitted patients in the ED. Part two of the hypothesis was that when InptOcc reaches a critical point, hypothesized a priori to be within the range 85-95\%, InptOcc has a statistically and operationally significant impact on LOS.

The method for regression was to identify whether there was a point at InptOcc (to use standard terminology, the "knot" in the regression line) [9] below which there was no significant relationship between InptOcc and LOS, but above which there was a significant association between InptOcc and LOS.

The initial step was generation of a scatterplot that assessed the relationship between InptOcc and the regression-predicted LOS. This graph, depicted in Figure 1, demonstrated the general location of the potential knot in the regression line: the graph depicts the tendency of the LOS to begin to rise sharply as a function of InptOcc when the latter starts to rise above $85-90 \%$.

The initial nonlinear model identified a statistically significant spline regression knot that matched the graphsuggested knot location, at $88.7 \%$ InptOcc (95\% CI, 86.590.9, $P<0.001)$. Below the InptOcc rate of $88.7 \%$ there was no significant association between InptOcc and LOC (95\% CI for association of -1.4 to $3.3, P=0.41$ ), but above the InptOcc rate of $88.7 \%$ the association was statistically significant $(P<0.001)$ and operationally substantial: at InptOcc above $88.7 \%$ each additional incremental percentage point in InptOcc was associated with 16 minutes longer LOS (95\% CI 12-20 minutes).

After the initial univariate analysis identified a statistically significant knot in the spline regression, potential confounders were included in the model as covariates. The covariate study period (as assessed in 50-day time frames over the 200-day study period) was found to be both nonsignificant $(P=0.52)$ and also to not be a confounder.

The covariates that were significant (by the likelihood ratio test) were day of week, ED census, percentage of higher-acuity cases, and admission percentage. In the model adjusting for these covariates, the point estimate for the effect of InptOcc on LOS was 13 minutes (95\% CI, 10 to $16, P<$ $0.001)$. In other words, for each percentage point increase in InptOcc above $88.7 \%$, there was a 13 -minute prolongation of LOS in the ED for admitted patients. The model's adjusted $r^{2}$ was 0.53 . The linear combination test to determine the difference in slopes for the InptOcc-LOS relationship below the knot versus above the knot was significant $(P<0.001)$.

\section{Discussion}

This study, although characterized by limitations to be addressed momentarily, provides some important information that can be useful in ED operations planning. Perhaps the most important lesson from the analysis is that mathematical techniques can be applied to operations data, in order to allow for data-driven decisions regarding resource allocation. The findings for the ED studied are less important than the demonstration that the techniques used were found beneficial; these techniques are easily applied to other EDs and are quite likely to be equally useful in a variety of settings.

It seems common sense that since increasing inpatient occupancy rates translates into fewer available inpatient beds, higher inpatient occupancy risks prolonged ED LOS for those patients in the ED waiting to be admitted to an open bed. The apparent truth of this relationship between InptOcc and LOS has led to a number of laudable efforts aimed at optimizing bed capacity control in the inpatient setting $[11,12]$.

The relationship between InptOcc and ED LOS is therefore previously characterized and relatively well-known. The new offering from the current study is the application of spline regression techniques to translate the commonsense understanding of the InptOcc-LOS relationship into mathematical terms that allow for closer examination. Such closer examination includes a search for whether or not there is an inflection point in the curve describing the relationship between decreasing available inpatient beds and increasing time spent by admitted patients in the ED.

The first finding of utility in the current study was that there was indeed a nonlinear association between InptOcc and LOS. This finding should not be too surprising; there is little reason to think that ranges of InptOcc still capable of accommodating patients admitted from the ED should impact ED LOS. But the common sense nature of the finding of nonlinearity of InptOcc-LOS leads to a more fundamental lesson for operations planners: there is indeed a 
point at which InptOcc starts to impact LOS in a substantial manner.

Operation stresses exist in even the best-run hospitals, especially during times of ED or hospital overcrowding. Planning for these times necessarily includes the ability to commit additional resources to help address the operational stress. Such planning has been described for myriad situations of hospital and ED operational stress, with an example being the literature describing "surge capacity" measures [13-15].

One point that is made consistently in the literature addressing operations of volume-overloaded EDs is as follows: finances are not unlimited and resources are increasingly constrained [15-17]. For example, problems with ED LOS could usually be met by simply adding large numbers of beds to the hospital's inpatient capability or by doubling the size of the ED itself. Solutions such as these are not financially viable, leading to a need to titrate response to operational stressors such that resources are allocated when needed, while being reserved when not needed. The identification of the point at which resources are "needed" marks the potential importance of this study.

On the other hand, waiting until the ED is in "crisis mode" before committing resources that can increase inpatient capacity (e.g., expedited discharges, calling in extra nurses to open inpatient beds) can be inefficient and ultimately costly (e.g., through increased LWBS). This study identified, at least for the study ED, the point at which resource allocation begins to make operational sense.

The advantage of translating the common sense notion of an InptOcc-LOS into mathematics is that such translation allows for a more precise pinpointing of the point at which rising InptOcc begins to be substantially problematic for LOS. In this study ED, the InptOcc level of $88 \%$ was identified as a candidate trigger point for hospital operations interventions. This occupancy level was actually less than that which was hypothesized by at least some of the operations personnel at the study hospital. Finding a "warning" level that was less than expected could potentially translate into more timely intervention and more successful prevention of prolongation of ED waiting times for admitted patients. Furthermore, even though there were only 200 days' data the $95 \%$ CI for the estimate of the location of the inflection point was reasonably small ( $87 \%$ to $91 \%)$.

Before discussing preliminary conclusions and potential next steps that will arise from this study, it is important to acknowledge numerous limitations to the methodology. These limitations do not negate the utility of the data analysis but they do relegate the inferences to a preliminary nature.

First, the data analysis was based on only 200 days of information. This was done for the straightforward reason that, prior to the commencement of this study's timeframe, the available information for the operational parameters was not reliably obtainable. Repeat analysis is planned for the future, when there are more data, but given the statistically significant findings in the reasonably narrow confidence intervals found in the various analyses the investigators do not believe that low study $n$ (i.e., number of days) was a substantial problem.
The second major limitation is that the study methodology did not adjust for changes on a time frame less than 24 hours. In other words, for each of the study days the parameters that were used in the model were the daily numbers rather than hour by hour data. For example, the daily ED census was used to adjust for how busy the department was, but of course there is variation within a day in the numbers of patients presenting to be seen; therefore, it is likely that the primary independent variable of interest (InptOcc) could have differential effects at different times of the day. Follow-up research is planned to "cone down" on data that are available in an hour-based (rather than a day-based) fashion to repeat the analyses reported in this study, but at this time reliable data on the smaller time frame are not available.

The third important limitation of this study is related to the previous limitation surrounding the time frame of data collection. In addition to the fact mentioned previously, regarding the use of data that represent summary statistics for a given day, there is an additional problem in that the study is using information that is not available at the time of a decision for resource allocation in an operationally stressed ED. It is true that the InptOcc variable, which was indeed the main focus of the study, is the most likely to be stable over a long enough time period to allow its use for real-time prediction of LOS problems (and thus allow InptOcc use for triggering resource allocation to stave off potential LOS problems). Other than the InptOcc variable, though, most of the other variables are not easily obtainable in a sufficiently real-time way to allow their application for on-the-spot decision-making about resource allocation. For example, while InptOcc is a relatively stable number over many hours during a given day, parameters such as the ED census and triage acuity (both of which were covariates in this study) are likely to change drastically over very short time periods. A maximally responsive model would incorporate these variables on an hour-by-hour basis, to provide the most useful predictive discrimination. On the other hand, this weakness is somewhat offset by this study's finding that at least one fairly stable parameter-InptOcc-was a significant and highly useful predictor of looming ED LOS problems.

An additional study limitation is that there are other variables that impact LOS that could not be included in this study model. This is demonstrated by the fact that the models adjusted $R$ squared indicated that this spline regression accounted for just over half of the variability in LOS; there are obviously some other factors in the mix for which the current model did not account. It is probable that parameters such as changing number (particularly on "shortstaffed" nursing days) and even identity of ED physicians and nurses influence LOS. It is also possible that technology issues, such as the electronic medical record, could impact LOS in a differential manner. These sorts of issues would seem more likely to bias the results toward a null finding, but it is important to keep in mind that future analyses should try and adjust for all parameters with the potential impact on LOS.

One final limitation of the study is related to the impact of the results. Even if the results are accepted as indicating a true association and inflection point for InptOcc-LOS, the study has merely identified a potential tool: use of an InptOcc 
"trigger" to execute operations interventions to improve ED flow. Application of the tool, with data-based analysis of the impact of application of the tool, must occur before any definitive conclusions can be drawn about the practicality of this technique for analysis of operations. Of course, if the study approach is determined to be valid, the results apply only to the ED from which data were drawn for the calculations. While the specific results are not generalizable, the potential utility of the use of spline regression and the application of the technique itself are quite generalizable and spelled out in detail in both statistical software manuals and related texts [9].

In conclusion, the results of the current study reproduced what is already known on this subject, that there is a statistically and operationally significant association between the percentage of acute inpatient hospital beds unoccupied and the LOS for ED patients who are admitted to the hospital. More importantly, the methodology we used for this study can be utilized by others to determine at what point a rise in their own inpatient hospital bed occupancy begins to significantly and operationally impact ED LOS. The identification of this inflection point on the InptOcc-LOS curve is theorized to provide a basis for triggering operations improvement of resource allocation to prevent ED LOS prolongation before it occurs. Follow-up studies are necessary to both better describe the relationship between InptOcc and LOS as well as determine whether the use of a particular InptOcc "trigger level" for operations improvement has the desired impact on ED efficiency and throughput.

\section{Conflict of Interests}

The authors declare that there is no conflict of interests regarding the publication of this paper.

\section{References}

[1] P. B. Patel, M. A. Combs, and D. R. Vinson, "Reduction of admit wait times: the effect of a leadership-based program," Academic Emergency Medicine, vol. 21, no. 3, pp. 266-273, 2014.

[2] S. W. Liu, S. H. Thomas, J. A. Gordon, A. G. Hamedani, and J. S. Weissman, "A pilot study examining undesirable events among emergency department-boarded patients awaiting inpatient beds," Annals of Emergency Medicine, vol. 54, no. 3, pp. 381-385, 2009.

[3] C. M. Sørup, P. Jacobsen, and J. L. Forberg, "Evaluation of emergency department performance-a systematic review on recommended performance and quality-in-care measures," Scandinavian Journal of Trauma, Resuscitation and Emergency Medicine, vol. 21, no. 1, article 62, 2013.

[4] M. Kennedy, C. E. MacBean, C. Brand, V. Sundararajan, and D. M. Taylor, "Review article: leaving the emergency department without being seen," Emergency Medicine Australasia, vol. 20, no. 4, pp. 306-313, 2008.

[5] B. H. Rowe, P. Channan, M. Bullard et al., "Characteristics of patients who leave emergency departments without being seen," Academic Emergency Medicine, vol. 13, no. 8, pp. 848-852, 2006.

[6] M. McHugh, K. J. Van Dyke, E. Howell, F. Adams, D. Moss, and J. Yonek, "Changes in patient flow among five hospitals participating in a learning collaborative," Journal for Healthcare Quality, vol. 35, pp. 21-29, 2013.

[7] V. W. Tsai, G. Q. Sharieff, J. T. Kanegaye, L. A. Carlson, and J. Harley, "Rapid medical assessment: improving pediatric emergency department time to provider, length of stay, and left without being seen rates," Pediatric Emergency Care, vol. 28, no. 4, pp. 354-356, 2012.

[8] S. K. Polevoi, J. Jewel Shim, C. E. McCulloch, B. Grimes, and P. Govindarajan, "Marked reduction in length of stay for patients with psychiatric emergencies after implementation of a comanagement model," Academic Emergency Medicine, vol. 20, no. 4, pp. 338-343, 2013.

[9] M. N. Mitchell, Interpreting and Visualizing Regression Models Using STATA, Stata Press, College Station, Tex, USA, 1st edition, 2012.

[10] D. Hosmer, S. Lemeshow, and R. Sturdivant, Applied Logistic Regression, John Wiley \& Sons, Hoboken, NJ, USA, 3rd edition, 2013.

[11] J. Jweinat, P. Damore, V. Morris, R. D’Aquila, S. Bacon, and T. J. Balcezak, "The safe patient flow initiative: a collaborative quality improvement journey at Yale-New Haven Hospital," Joint Commission Journal on Quality and Patient Safety/Joint Commission Resources, vol. 39, pp. 447-459, 2013.

[12] B. Ortiga, A. Salazar, A. Jovell, J. Escarrabill, G. Marca, and $\mathrm{X}$. Corbella, "Standardizing admission and discharge processes to improve patient flow: a cross sectional study," BMC Health Services Research, vol. 12, no. 1, article 180, 2012.

[13] M. Abir, M. M. Davis, P. Sankar, A. C. Wong, and S. C. Wang, "Design of a model to predict surge capacity bottlenecks for burn mass casualties at a large academic medical center," Prehospital and Disaster Medicine, vol. 28, no. 1, pp. 23-32, 2013.

[14] R. D. Kearns, K. M. Conlon, A. L. Valenta et al., "Disaster planning: the basics of creating a burn mass casualty disaster plan for a burn center," Journal of Burn Care and Research, vol. 35, no. 1, pp. el-e13, 2013.

[15] S. Braithwaite, B. Friedman, R. Mutter, and M. Handrigan, "Microsimulation of financial impact of demand surge on hospitals: the H1N1 influenza pandemic of fall 2009," Health Services Research, vol. 48, no. 2, pp. 735-752, 2013.

[16] C. W. Baugh, A. K. Venkatesh, and J. S. Bohan, "Emergency department observation units: a clinical and financial benefit for hospitals," Health Care Management Review, vol. 36, no. 1, pp. 28-37, 2011.

[17] D. A. Bradt, P. Aitken, G. FitzGerald, R. Swift, G. O’Reilly, and B. Bartley, "Emergency department surge capacity: reco mendations of the Australasian surge strategy working group," Academic Emergency Medicine, vol. 16, no. 12, pp. 1350-1358, 2009. 


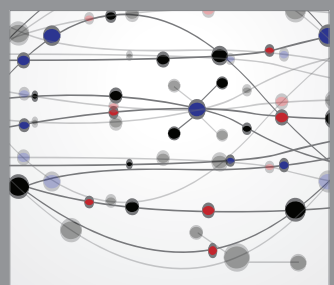

The Scientific World Journal
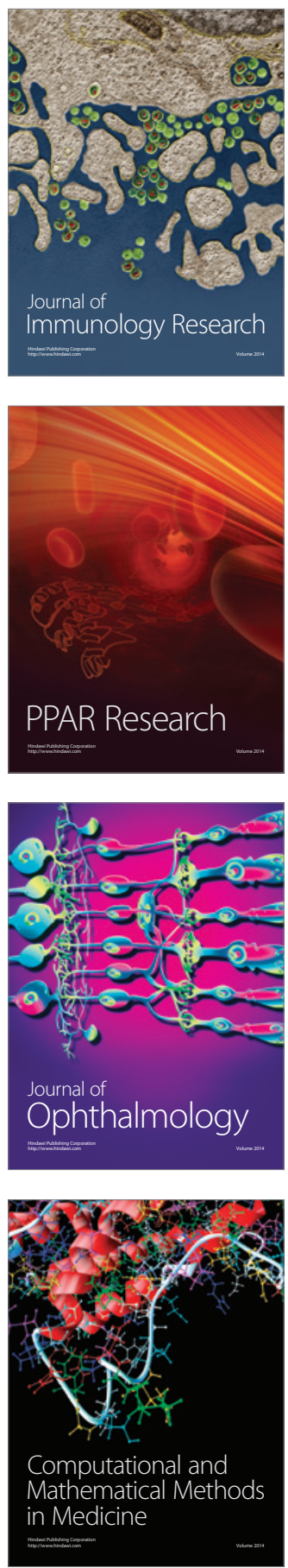

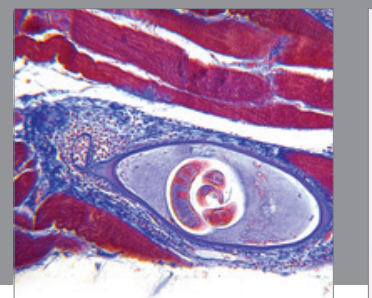

Gastroenterology

Research and Practice
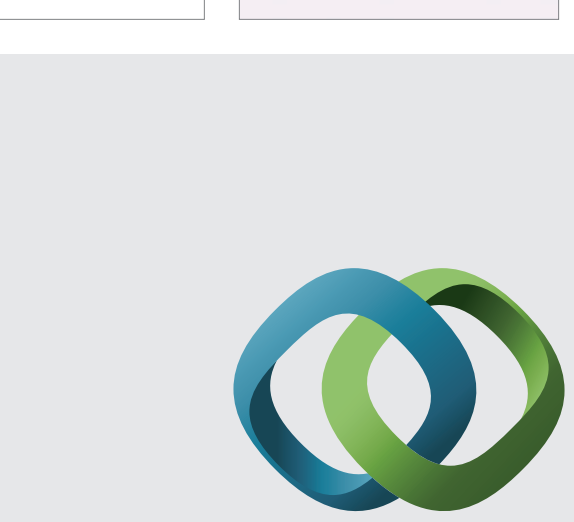

\section{Hindawi}

Submit your manuscripts at

http://www.hindawi.com
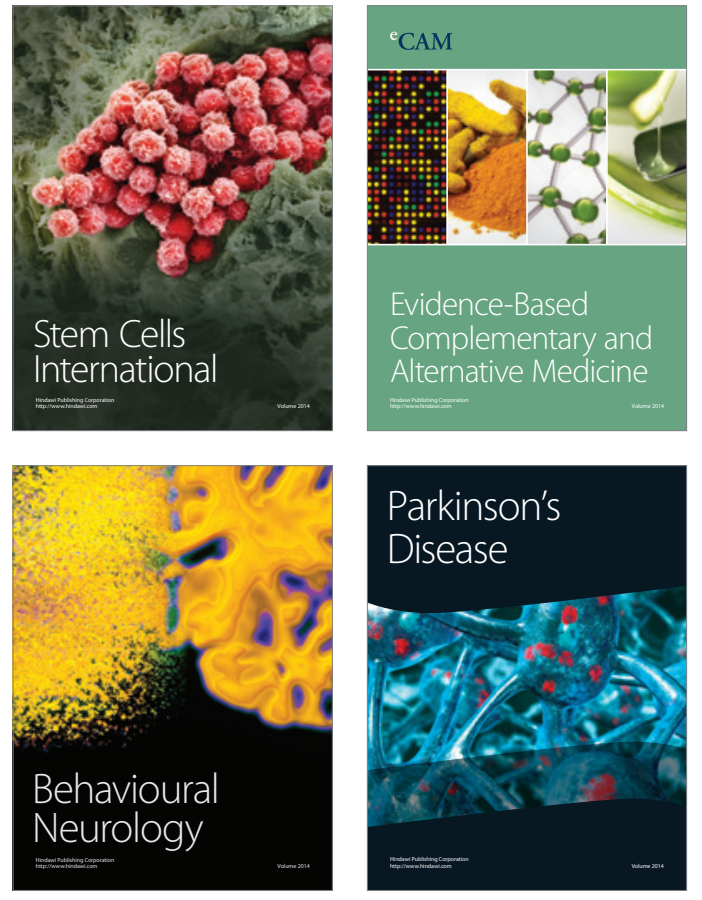
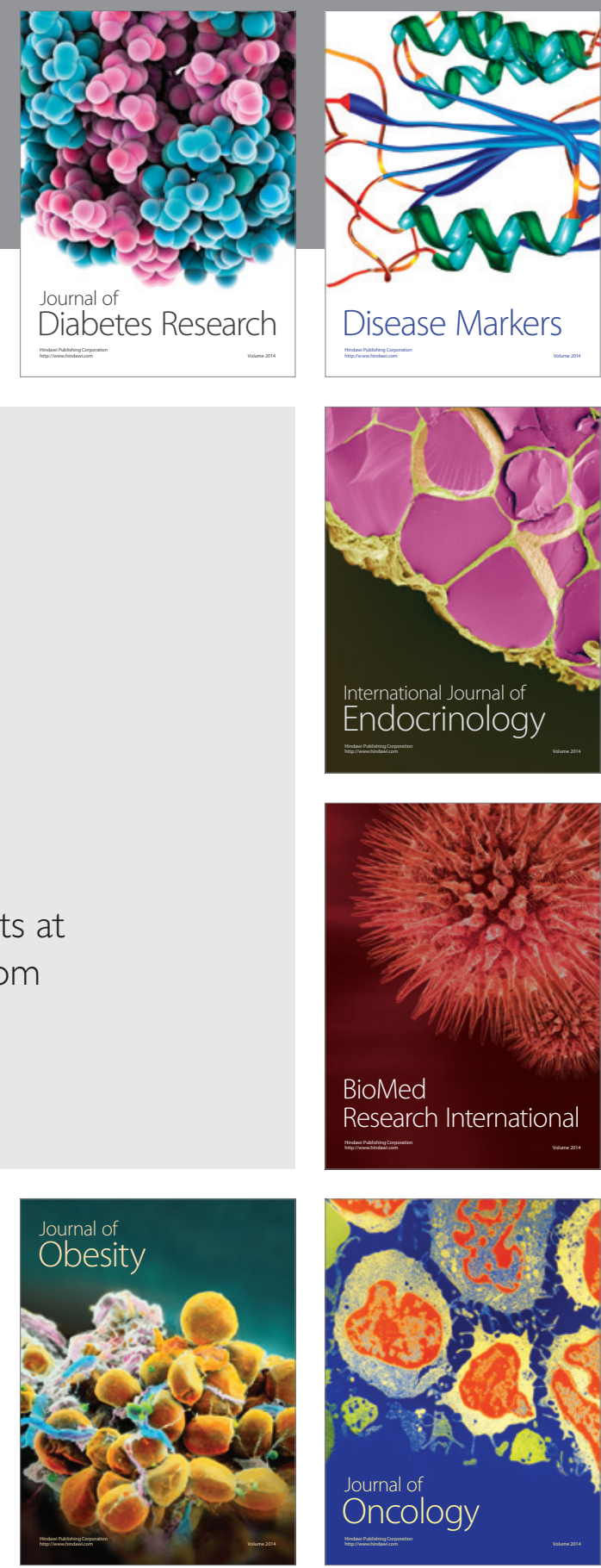

Disease Markers
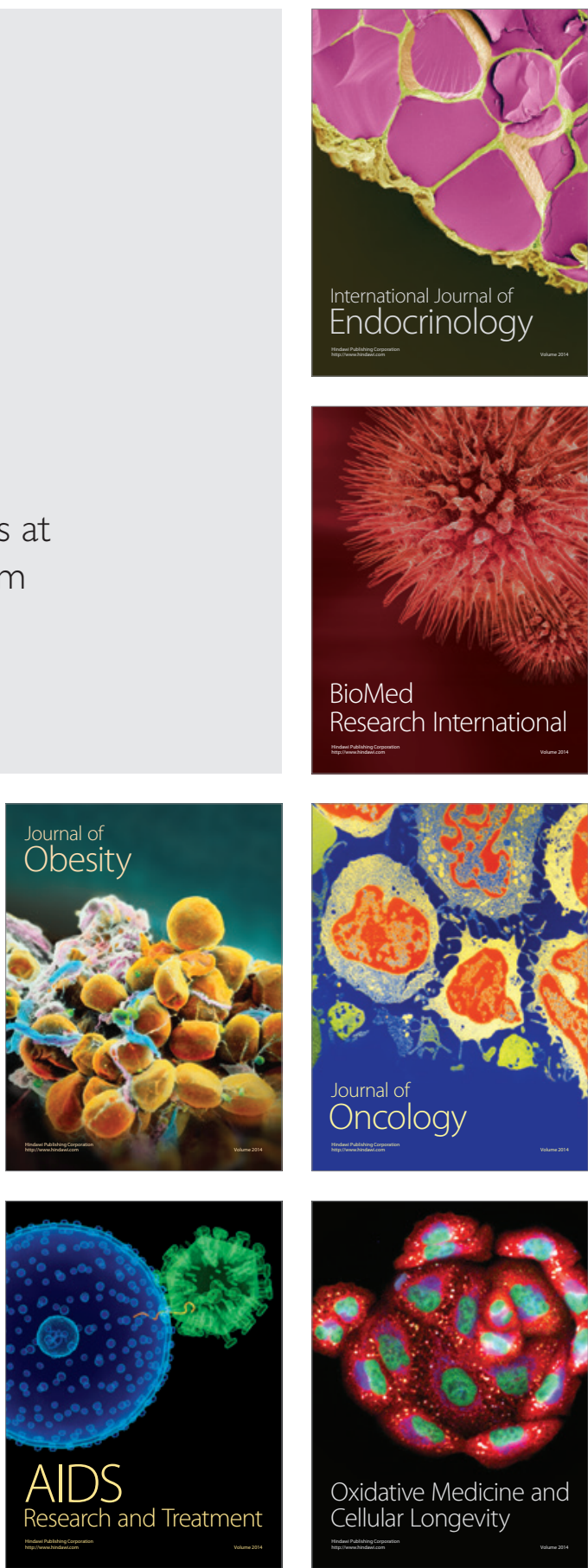